\title{
AN ACCURACY-TYPE ORDER ON ROUGH SETS
}

\author{
ATTILA KÖREI, SÁNDOR RADELECZKI, AND SZILVIA SZILÁGYI
}

Received 28 April, 2019

\begin{abstract}
We introduce a new order on rough sets inspired by the notion of accuracy measure. Rough sets ordered by this accuracy-type order form a complete lattice, which is isomorphic to the lattice where rough sets are ordered traditionally by the inclusion relation. Although the lattices are order-isomorphic, the elements can be totally differently located in them. The isomorphy of the lattices is verified by introducing the so-called boundary representation of rough sets. Some basic properties of the introduced order are also presented and an illustrative example demonstrates the relationship between the different structures.
\end{abstract}

2010 Mathematics Subject Classification: 06B15; 68T37; 06D15; 06D30

Keywords: lower and upper approximation, rough sets, accuracy measure, boundary region, Stone lattice

\section{INTRODUCTION}

In this paper we examine different orders which can be defined on rough sets. The notion of rough sets were introduced by Pawlak in [8] aiming an extension of standard set theory. In his original model, Pawlak assumed that our knowledge about the objects of a universe $U$ is given in terms of an equivalence relation $E \subseteq U \times U$ reflecting the indiscernibility of the objects. Two elements of $U$ are $E$-equivalent if we cannot distinguish them by their attributes known by us. Using the classes of this equivalence for any subset of the object set a pair of sets called lower and upper approximations is defined. These pairs may be identified with rough sets and ordering them by the component-wise inclusion, we obtain a well-known complete lattice, the rough set lattice, whose properties were investigated in details. Forming the ratio of the cardinalities of the lower and upper approximations we obtain the socalled accuracy measure which is a basic numerical characterization of rough sets. This measure and its improved versions play an important role in granular computing and were studied in different papers [10-14]. Inspired by the accuracy measure we propose a new order on rough sets. Rough sets ordered by this accuracy-type order form a complete lattice which is isomorphic to the lattice obtained by the traditional inclusion order, although the sets can be completely differently positioned in this new lattice. Our work was also motivated by the papers $[1,6]$. In [6] an extension 
of the classical set algebra is presented, which can be considered as a counterpart of Belnap's four-valued logic. It is known that Belnap's four-valued logic deals with two truth values [1]: one of them describes the incompleteness of the information, and the other expresses its consistency.

\section{PRELIMINARIES}

For any equivalence relation $E \subseteq U \times U$ and any element $u \in U$, let $E(u)$ denote the $E$-equivalence class of $u$, i.e.

$$
E(u):=\{x \in U \mid(u, x) \in E\} .
$$

Then for any subset $X \subseteq U$ the lower approximation of $X$ with respect to $E$ is defined as

$$
X_{E}:=\{x \in U \mid E(x) \subseteq X\},
$$

and the upper approximation of $X$ is given by

$$
X^{E}:=\{x \in U \mid E(x) \cap X \neq \varnothing\} .
$$

The set $\mathrm{BN}_{E}(X):=X^{E} \backslash X_{E}$ will be referred to as the $E$-boundary region of $X$. Since $x \in E(x)$ for each $x \in U$, we get that $X_{E} \subseteq X \subseteq X^{E}$ and hence we can partition the elements of $U$ into three disjoint classes with respect to the set $X$ (see [5]):

(1) The elements which are certainly in $X$. These elements are interpreted as the elements of $X_{E}$, because if $x \in X_{E}$, then all the elements of the equivalence class $E(x)$ of $x$ are in $X$.

(2) The elements which are certainly not in $X$. The elements $x$ of $U$ such that all the elements of the class $E(x)$ of $x$ are not in $X: E(x) \cap X=\varnothing$.

(3) The elements which are possibly in $X$, i.e. $x \in U$ which are $E$-related at least with one element of $X$ and also with at least one element from its complement $X^{c}=U \backslash X$. In other words, now $E(x) \cap X \neq \varnothing$ and $E(x) \nsubseteq X$, i.e. $x \in$ $X^{E} \backslash X_{E}=\mathrm{BN}_{E}(X)$.

If $X_{E}=X^{E}$, i.e. the boundary region of $X$ is the empty set $\varnothing$; then the set $X$ is called exact with respect to $E$. Now let $X, Y \subseteq U$. The following properties will be used in our paper:

(a) $\varnothing^{E}=\varnothing_{E}=\varnothing, U^{E}=U_{E}=U$;

(b) $\left(X_{E}\right)^{E}=\left(X_{E}\right)_{E}=X_{E},\left(X^{E}\right)^{E}=\left(X^{E}\right)_{E}=X^{E}$;

(c) If $X \subseteq Y$ then $X_{E} \subseteq Y_{E}$ and $X^{E} \subseteq Y^{E}$.

By definition, for any set $X \subseteq U, X_{E}, X^{E}$ and also $\mathrm{BN}_{E}(X)$ are unions of some classes of the equivalence $E$. In what follows, unions of $E$-equivalence classes will be called definable sets and their set will be denoted by $\operatorname{DF}(U, E)$. Thus

$$
\operatorname{DF}(U, E)=\{X \subseteq U \mid X=\bigcup\{E(x) \mid x \in X\} .
$$


As for each $X \in \mathrm{DF}(U, E), X^{E}=X_{E}=X$, according to (b), we deduce that $\operatorname{DF}(U, E),\left\{X^{E} \mid X \subseteq U\right\}$ and $\left\{X_{E} \mid X \subseteq U\right\}$ are the same systems of sets, and the definable sets are just the exact sets with respect to $E$. Clearly, with respect to $\subseteq$ they form an ordered set $(\mathrm{DF}(U, E), \subseteq)$. It is known that $(\mathrm{DF}(U, E), \subseteq)$ is a complete Boolean lattice, where the lattice operations $\vee$ and $\wedge$ are just the set-theoretical union and intersection of the definable sets. Since the set-theoretical complement $X^{c}$ of a set $X \in \mathrm{DF}(U, E)$ is also definable, the complement of $X$ in the lattice $(\mathrm{DF}(U, E), \subseteq)$ is just $X^{c}$.

The rough set of $X$ can be identified with the pair $\left(X_{E}, X^{E}\right)$ and the set of all rough sets of $E$ is usually given as

$$
R S(U, E):=\left\{\left(X_{E}, X^{E}\right) \mid X \subseteq U\right\} .
$$

In other words, with every rough set we associate two exact sets, i.e. the lower approximation $X_{E}$ and the upper approximation $X^{E}$ of the target set $X . X_{E}$ is also called the positive region of $X$, while $\left(X^{E}\right)^{c}$ the negative region of $X$. The set $R S(U, E)$ may be canonically ordered by the component-wise inclusion (called inclusion order):

$$
\left(X_{E}, X^{E}\right) \leq\left(Y_{E}, Y^{E}\right) \Leftrightarrow X_{E} \subseteq Y_{E} \text { and } X^{E} \subseteq Y^{E},
$$

obtaining a partially ordered set $\mathbb{R} \$(U, E):=(R S(U, E), \leq)$. It is well-known that $\mathbb{R} \$(U, E)$ is a completely distributive Stone lattice (see e.g. [9]) with the least element $(\varnothing, \varnothing)$ and the greatest element $(U, U)$. (Later this result was improved by $\mathrm{S}$. D. Comer [2] by showing that $\mathbb{R} \$(U, E)$ is a so-called regular double Stone algebra). Of course, a rough set can be characterized not only by the fact that how "wide" are the sets $X_{E}$ and $X^{E}$ assigned to it, but also by the fact that how "vague" is the rough set itself, i.e. how wide is its boundary with respect to its lower or upper approximation. For instance, a (finite) rough set different from $(\varnothing, \varnothing)$ can also be characterized numerically by the coefficient:

$$
\alpha_{E}(X)=\frac{\left|X_{E}\right|}{\left|X^{E}\right|}=1-\frac{\left|\mathrm{BN}_{E}(X)\right|}{\left|X_{E}\right|+\left|\mathrm{BN}_{E}(X)\right|}
$$

called the accuracy of approximation, where $|X|$ denotes the cardinality of the set $X \neq \varnothing$. For $\varnothing$ we define $\alpha_{E}(\varnothing)=1$. Inspired by this notion introduced by Z. Pawlak in [8], we define a new partial order $\sqsubseteq$ on the set $R S(U, E)$ as follows:

$$
\left(X_{E}, X^{E}\right) \sqsubseteq\left(Y_{E}, Y^{E}\right) \Leftrightarrow X_{E} \subseteq Y_{E} \text { and } \mathrm{BN}_{E}(Y) \subseteq \mathrm{BN}_{E}(X) .
$$

Clearly, $\sqsubseteq$ is a reflexive and transitive relation. Let $\left(X_{E}, X^{E}\right) \sqsubseteq\left(Y_{E}, Y^{E}\right)$ and $\left(Y_{E}, Y^{E}\right) \sqsubseteq\left(X_{E}, X^{E}\right)$. Then $X_{E}=Y_{E}$ and $\mathrm{BN}_{E}(Y)=\mathrm{BN}_{E}(X)$ together imply

$$
X^{E}=X_{E} \cup \mathrm{BN}_{E}(X)=Y_{E} \cup \mathrm{BN}_{E}(Y)=Y^{E},
$$

whence we get

$$
\left(X_{E}, X^{E}\right)=\left(Y_{E}, Y^{E}\right)
$$


This proves that $\sqsubseteq$ is also antisymmetric, thus it is a partial order.

On the other hand, if $\left(X_{E}, X^{E}\right)$ and $\left(Y_{E}, Y^{E}\right)$ are rough sets different from $(\varnothing, \varnothing)$ and $\left(X_{E}, X^{E}\right) \sqsubseteq\left(Y_{E}, Y^{E}\right)$, then $\left|X_{E}\right| \leq\left|Y_{E}\right|$ and $\left|\mathrm{BN}_{E}(X)\right| \geq\left|\mathrm{BN}_{E}(Y)\right|$ imply

and hence

$$
\frac{\left|X_{E}\right|}{\left|\mathrm{BN}_{E}(X)\right|}+1 \leq \frac{\left|Y_{E}\right|}{\left|\mathrm{BN}_{E}(Y)\right|}+1
$$

$$
\alpha_{E}(X)=1-\frac{1}{\frac{\left|X_{E}\right|}{\left|\mathrm{BN}_{E}(X)\right|}+1} \leq 1-\frac{1}{\frac{\left|Y_{E}\right|}{\left|\mathrm{BN}_{E}(Y)\right|}+1}=\alpha_{E}(Y) .
$$

We also note that the accuracy measure can be generated using the MarczewskiSteinhaus metric defined on the subsets of a universe (see $[12,13])$.

In what follows, we will study the partially ordered set $(R S(U, E)$, $\sqsubseteq)$. Comparing two rough sets $\left(A_{E}, A^{E}\right)$ and $\left(B_{E}, B^{E}\right)$ by the relation $\sqsubseteq$, the inclusion $A_{E} \subseteq B_{E}$ expresses that the positive region of the first rough set is less than the positive region of the second one, i.e. the truth-value represented by the second one is bigger than the truth-value of the first one. The relation $\mathrm{BN}_{E}(B) \subseteq \mathrm{BN}_{E}(A)$ expresses that the second rough set is closer to an exact set than the first one, i.e. the information in the second one is more consistent than in the first one. Surprisingly, although $\sqsubseteq$ is significantly different from the inclusion order, $(R S(U, E)$, 드) is isomorphic to the lattice $(R S(U, E), \leq)$. To understand the properties of the lattice $(R S(U, E)$, $\sqsubseteq)$ we need some more notions.

\section{Lattice theoretical notions}

A complete lattice $L$ is called completely distributive (see e.g. [3]) if for any doubly indexed family of elements $\left\{x_{i, j}\right\}_{i \in I, j \in J},(I, J \neq \varnothing)$ we have

$$
\bigwedge_{i \in I}\left(\bigvee_{j \in J} x_{i, j}\right)=\bigvee_{f: I \rightarrow J}\left(\bigwedge_{i \in I} x_{i, f(i)}\right)
$$

For instance, any complete Boolean lattice and finite distributive lattice is completely distributive. A bounded lattice $L$ is called pseudocomplemented, if for any $x \in L$ there exists an element $x^{*} \in L$ such that $x \wedge y=0$, for some $y \in L \Leftrightarrow y \leq x^{*}$. A bounded distributive lattice $L$ is called a Stone lattice, whenever the equality

$$
x^{*} \vee x^{* *}=1
$$

holds for all $x \in L$ (see [4]).

A bijective function $\phi: M \rightarrow N$ between two ordered sets $\left(M_{1}, \leq_{1}\right)$ and $\left(M_{2}, \leq_{2}\right)$ is called an order isomorphism if for every $x$ and $y$ in $M_{1}$

$$
x \leq_{1} y \Leftrightarrow \phi(x) \leq_{2} \phi(y) .
$$

Specially, if $\phi$ is an order isomorphism from $M_{1}$ to $M_{2}$ where $\left(M_{1}, \leq_{1}\right)$ and $\left(M_{2}, \leq_{2}\right.$ ) are complete lattices, then $\phi$ preserves the lattice operations. 
A singleton of a binary relation $E \subseteq U \times U$ is an element $s \in U$ such that $E(s)=$ $\{s\}$. The set of singletons will be denoted by $S(E)$, or simply by $S$, when there is no danger of confusion. Let $E \subseteq U \times U$ be an equivalence. Then clearly, for any $s \in S$ we have $\{s\}_{E}=\{s\}^{E}=\{s\}$, whence $S_{E}=S^{E}=S$. Thus $S \in \operatorname{DF}(U, E)$. It was proved in [7] that for any $X, Y \in \mathrm{DF}(U, E)$ with $X \subseteq Y$, the pair $(X, Y)$ is a rough set defined by $E$ if and only if $Y \backslash X$ does not contain any singleton of $E$. Since $Y$ is a disjoint union of $X$ and $Y \backslash X$, the latter condition is equivalent to $X \cap S=Y \cap S$. In other words,

$$
(X, Y) \in R S(U, E) \Leftrightarrow X, Y \in \mathrm{DF}(U, E), X \subseteq Y \text { and } X \cap S=Y \cap S .
$$

Therefore, the rough sets of $E$ can be identified with the set

$$
R S(U, E)=\left\{(X, Y) \in \operatorname{DF}(U, E)^{2} \mid X \subseteq Y \text { and } X \cap S=Y \cap S\right\} .
$$

Since the operations in the lattice $\mathbb{R} \$(U, E)=(R S(U, E), \leq)$ are defined as the component-wise union and intersection of the pairs of definable sets, $\mathbb{R} \$(U, E)$ constitute a complete sublattice of the direct square $(\operatorname{DF}(U, E), \subseteq) \times$ $(\mathrm{DF}(U, E), \subseteq)$. The representation given in (2.2) is usually called increasing representation of rough sets induced by $E$.

Disjoint representations of rough sets were introduced by P. Pagliani in [5]. Each rough set $\left(X_{E}, X^{E}\right)$ may be represented as a pair $\left(X_{E},\left(X^{E}\right)^{c}\right)$, called the disjoint rough set of $X$. Clearly, $\left(X_{E},\left(X^{E}\right)^{c}\right.$ belongs to $\operatorname{DF}(U, E)^{2}$, and now $\left(X^{E}\right)^{c}$ can be interpreted as the set of elements that certainly are outside of $X$. (This is the negative region of $X$.) We denote

$$
d R S(U, E):=\left\{\left(X_{E},\left(X^{E}\right)^{c}\right) \mid X \subseteq U\right\} .
$$

It was proved in [5] that

$$
d R S(U, E)=\left\{(A, B) \in \operatorname{DF}(U, E)^{2} \mid A \cap B=\varnothing, S \subseteq A \cup B\right\} .
$$

The pairs $(A, B),(C, D) \in d R S(U, E)$ are ordered as follows:

$$
(A, B) \lesssim(C, D) \Leftrightarrow(A \subseteq C \text { and } B \supseteq D)
$$

In [5] it was shown that $\sigma:(A, B) \rightarrow\left(A, B^{c}\right)$ is an order-isomorphism between $(R S(U, E), \leq)$ and $(d R S(U, E), \lesssim)$, which allows us to call (3) the disjoint representation of rough sets induced by $E$.

\section{BOUNDARY REPRESENTATION OF ROUGH SETS}

Let us observe first that the rough set corresponding to a reference set $X \subseteq U$ can be determined not only by its lower and upper approximations $X_{E}$ and $X^{E}$, but also by $X_{E}$ and its boundary $\mathrm{BN}_{E}(X)=X^{E} \backslash X_{E} \in \mathrm{DF}(U, E)$. Indeed, we have $X^{E}=X_{E} \cup \mathrm{BN}_{E}(X)$ and $X_{E} \cap \mathrm{BN}_{E}(X)=\varnothing, S \cap \mathrm{BN}_{E}(X)=\varnothing$. In fact, we have the following 
Lemma 1. Let $E \subseteq U \times U$ be an equivalence relation and $S$ be the set of its singletons. Then for any $A, D \in \operatorname{DF}(U, E)$, the pair $(A, A \cup D) \in R S(U, E)$ if and only if $(A \cup S) \cap D=\varnothing$.

Proof. If $(A, A \cup D) \in R S(U, E)$, then $(A, A \cup D)=\left(X_{E}, X^{E}\right)$, for some $X \subseteq U$ and hence

$$
D=(A \cup D) \backslash A=X^{E} \backslash X_{E}=\mathrm{BN}_{E}(X) .
$$

Then $A \cap D=\varnothing$ and

$$
S \cap D=S \cap \mathrm{BN}_{E}(X)=\varnothing
$$

imply $(A \cup S) \cap D=\varnothing$.

Conversely, assume that $(A \cup S) \cap D=\varnothing$. Then $S \cap D=\varnothing$. Since $A, A \cup D \in$ $\mathrm{DF}(U, E), A \subseteq A \cup D$ and

$$
(A \cup D) \cap S=(A \cap S) \cap(D \cap S)=A \cap S,
$$

in view of (2.1) we obtain that $(A, A \cup D) \in R S(U, E)$.

As an immediate corollary we obtain:

Corollary 1. For any equivalence $E \subseteq U \times U$,

$$
R S(U, E)=\{(A, A \cup D) \mid A, D \in \operatorname{DF}(U, E) \text {, and }(A \cup S) \cap D=\varnothing\} .
$$

Now, let us consider the following set

$$
B R S(U, E)=\{(A, D) \mid A, D \in \mathrm{DF}(U, E) \text {, and }(A \cup S) \cap D=\varnothing\} .
$$

Defining on $B R S(U, E)$ a partial order as follows:

$$
\left(A_{1}, D_{1}\right) \unlhd\left(A_{2}, D_{2}\right) \Longleftrightarrow A_{1} \subseteq A_{2} \text { and } D_{2} \subseteq D_{1},
$$

we obtain a partially ordered set $\operatorname{BR} \$(U, E):=(B R S(U, E), \unlhd)$. Since we will prove that $\mathbb{B} \mathbb{R} \$(U, E)$ is isomorphic to the lattice $\mathbb{R} \$(U, E)$, we can call $\mathbb{B} \mathbb{R} \$(U, E)$ boundary representation of the rough sets of $E \subseteq U \times U$. We can see that this is closely related to the disjoint representation of rough sets.

Theorem 1. Let $E$ be an arbitrary equivalence on the universe $U$. Then $\mathbb{B} \mathbb{R} \$(U, E)$ and $\mathbb{R} \$(U, E)$ are order-isomorphic.

Proof. Let us consider the maps

$$
\varphi: R S(U, E) \rightarrow B R S(U, E), \quad \varphi(A, B)=\left(A,(B \cup S)^{c}\right),
$$

for all $(A, B) \in R S(U, E)$ and

$$
\psi: B R S(U, E) \rightarrow R S(U, E), \quad \psi(A, D)=\left(A, A \cup(D \cup S)^{c}\right),
$$

for all $(A, D) \in B R S(U, E)$.

First, check that $\varphi$ and $\psi$ are well-defined: Indeed, for any $(A, B) \in R S(U, E)$ we have $A, B \cup S \in \mathrm{DF}(U, E)$, whence $(B \cup S)^{c} \in \mathrm{DF}(U, E)$ and

$$
(A \cup S) \cap(B \cup S)^{c}=(A \cup S) \cap B^{c} \cap S^{c}=\left(A \cap B^{c} \cap S^{c}\right) \cup\left(S \cap B^{c} \cap S^{c}\right)=\varnothing,
$$


because $S \cap S^{c}=\varnothing$, and $A \subseteq B$ implies $A \cap B^{c}=\varnothing$. Hence $\left(A,(B \cup S)^{c}\right) \in$ $B R S(U, E)$, according to (2). Further, for each $(A, D) \in B R S(U, E), A, D, S \in$ $\mathrm{DF}(U, E)$ yields $A \cup(D \cup S)^{c} \in \mathrm{DF}(U, E)$. Clearly, $A \subseteq A \cup(D \cup S)^{c}$ and

$$
\begin{aligned}
\left(A \cup(D \cup S)^{c}\right) \cap S=\left(A \cup\left(D^{c} \cap S^{c}\right)\right) \cap S=(A \cap S) \cup\left(D^{c} \cap S^{c} \cap S\right) & \\
& =(A \cap S) \cup \varnothing=A \cap S .
\end{aligned}
$$

Hence $\left(A, A \cup(D \cup S)^{c}\right) \in R S(U, E)$.

It is easy to see that both $\varphi$ and $\psi$ are order-preserving. Indeed, for some $\left(A_{1}, B_{1}\right)$, $\left(A_{2}, B_{2}\right) \in R S(U, E)$ let $\left(A_{1}, B_{1}\right) \leq\left(A_{2}, B_{2}\right)$. Then $A_{1} \subseteq A_{2}$ and $B_{1} \cup S \subseteq B_{2} \cup S$, and hence $\left(B_{2} \cup S\right)^{c} \subseteq\left(B_{1} \cup S\right)^{c}$. This implies

$$
\varphi\left(A_{1}, B_{1}\right)=\left(A_{1},\left(B_{1} \cup S\right)^{c}\right) \unlhd\left(A_{2},\left(B_{2} \cup S\right)^{c}\right)=\varphi\left(A_{2}, B_{2}\right) .
$$

Similarly, let $\left(A_{1}, D_{1}\right) \unlhd\left(A_{2}, D_{2}\right)$ for some $\left(A_{1}, D_{1}\right),\left(A_{2}, D_{2}\right) \in B R S(U, E)$. Then $A_{1} \subseteq A_{2}$ and $D_{2} \subseteq D_{1}$ yields $\left(D_{1} \cup S\right)^{c} \subseteq\left(D_{2} \cup S\right)^{c}$, and hence we get

$$
\psi\left(A_{1}, D_{1}\right)=\left(A_{1},\left(D_{1} \cup S\right)^{c}\right) \leq\left(A_{2},\left(D_{2} \cup S\right)^{c}\right)=\psi\left(A_{2}, D_{2}\right) .
$$

Finally, observe that the mappings $\varphi$ and $\psi$ are inverses each of other. Indeed, for any $(A, D) \in B R S(U, E)$ we have

$$
\begin{gathered}
\varphi(\psi(A, D))=\varphi\left(A, A \cup(D \cup S)^{c}\right)=\left(A,\left(A \cup(D \cup S)^{c} \cup S\right)^{c}\right) \\
=\left(A, A^{c} \cap(D \cup S) \cap S^{c}\right)=\left(A,\left(A^{c} \cap D \cap S^{c}\right) \cup\left(A^{c} \cap S \cap S^{c}\right)\right) \\
=\left(A,(A \cup S)^{c} \cap D\right)=(A, D),
\end{gathered}
$$

because $(A \cup S) \cap D=\varnothing$ implies $D \subseteq(A \cup S)^{c}$, i.e. $(A \cup S)^{c} \cap D=D$.

On the other hand, let $(A, B) \in R S(U, E)$. Then

$$
\begin{gathered}
\psi(\varphi(A, B))=\psi\left(A,(B \cup S)^{c}\right)=\left(A, A \cup\left[(B \cup S)^{c} \cup S\right]^{c}\right) \\
=\left(A, A \cup\left[(B \cup S) \cap S^{c}\right]\right)=\left(A, A \cup\left[\left(B \cap S^{c}\right) \cup\left(S \cap S^{c}\right)\right]\right) \\
=\left(A, A \cup\left(B \cap S^{c}\right)\right)=(A, B) .
\end{gathered}
$$

The last equality holds because

$$
B=B \cap\left(S \cup S^{c}\right)=(B \cap S) \cup\left(B \cap S^{c}\right)=(A \cap S) \cup\left(B \cap S^{c}\right)
$$

implies

$$
A \cup\left(B \cap S^{c}\right)=A \cup(A \cap S) \cup\left(B \cap S^{c}\right)=A \cup B=B .
$$

The above results show that $\varphi$ and $\psi$ are order-isomorphism, hence the ordered sets $\mathbb{B} \mathbb{R} \Phi(U, E)$ and $\mathbb{R} \Phi(U, E)$ are isomorphic.

Now let us consider the partially ordered set $(R S(U, E)$, $\subseteq)$ defined by the order $\sqsubseteq$. Now we are able to prove

Proposition 1. Let $E$ be an equivalence on the universe $U$. Then the partially ordered sets $(R S(U, E), \sqsubseteq)$ and $(R S(U, E), \leq)$ are order-isomorphic. 
Proof. Observe that it is enough to prove that $(R S(U, E), \sqsubseteq)$ and $(B R S(U, E), \unlhd)$ are order-isomorphic. Then Theorem 1 will imply that $(R S(U, E), \sqsubseteq) \cong(R S(U, E), \leq)$.

Let us consider the maps for all $\left(A_{E}, A^{E}\right) \in R S(U, E)$

$$
f: R S(U, E) \rightarrow B R S(U, E), \quad f\left(A_{E}, A^{E}\right)=\left(A_{E}, A^{E} \backslash A_{E}\right),
$$

and for all $(A, D) \in B R S(U, E)$

$$
g: B R S(U, E) \rightarrow R S(U, E), \quad g(A, D)=(A, A \cup D) .
$$

Since $A_{E}, A^{E} \backslash A_{E} \in \operatorname{DF}(U, E)$ and

$$
\left(A_{E} \cup S\right) \cap\left(A^{E} \backslash A_{E}\right)=\left(A_{E} \cup S\right) \cap \mathrm{BN}_{E}(A)=\varnothing,
$$

we have $\left(A_{E}, A^{E} \backslash A_{E}\right) \in B R S(U, E)$, and hence $f$ is well-defined. Observe also that for any $(A, D) \in B R S(U, E)$, we have $(A, A \cup D) \in R S(U, E)$. Indeed,

$$
(A \cup S) \cap D=\varnothing
$$

by definition of $B R S(U, E)$, and hence Lemma 1 yields $(A, A \cup D) \in R S(U, E)$. Thus the map $g$ is also well-defined. Since

$$
f(g(A, D))=f(A, A \cup D)=(A,(A \cup D) \backslash A)=(A, D),
$$

and

$$
g\left(f\left(A_{E}, A^{E}\right)\right)=g\left(A_{E}, A^{E} \backslash A_{E}\right)=\left(A_{E}, A^{E}\right),
$$

for all $(A, D) \in B R S(U, E)$, and $\left(A_{E}, A^{E}\right) \in R S(U, E)$, the maps $f$ and $g$ are inverses each of other. Therefore $f$ and $g$ are bijective mappings.

Now let $\left(X_{E}, X^{E}\right),\left(Y_{E}, Y^{E}\right) \in R S(U, E)$. Then we can write:

$$
\begin{aligned}
\left(X_{E}, X^{E}\right) \sqsubseteq\left(Y_{E}, Y^{E}\right) & \Leftrightarrow X_{E} \subseteq Y_{E} \text { and } Y^{E} \backslash Y^{E} \subseteq X^{E} \backslash X_{E} \\
& \Leftrightarrow\left(X_{E}, X^{E} \backslash X_{E}\right) \unlhd\left(Y_{E}, Y^{E} \backslash Y_{E}\right) \\
& \Leftrightarrow f\left(X_{E}, X^{E}\right) \unlhd f\left(Y_{E}, Y^{E}\right) .
\end{aligned}
$$

This proves that $f$ (and also its inverse $g$ ) is an order-isomorphism.

Corollary 2. Let $E \subseteq U \times U$ be an equivalence relation. Then $(B R S(U, E), \unlhd)$ and $(R S(U, E), \sqsubseteq)$ are completely distributive Stone lattices.

It is not hard to determine the form of the join and meet operation in the new lattice $(R S(U, E), \sqsubseteq)$.

Proposition 2. Let $E \subseteq U \times U$ be an equivalence relation. Then for any system $\mathscr{H} \subseteq \mathcal{P}(U)$ and $\left(X_{E}, X^{E}\right) \in R S(U, E), X \in \mathscr{H}$, the join and meet in the complete lattice $(R S(U, E)$, $\subseteq)$ are given by the formulas:

$$
\bigsqcup_{X \in \mathscr{H}}\left(X_{E}, X^{E}\right)=\left(\bigcup_{X \in \mathscr{H}} X_{E},\left(\bigcup_{X \in \mathscr{H}} X_{E}\right) \bigcup\left(\bigcap_{X \in \mathscr{H}} B N_{E}(X)\right)\right),
$$




$$
\prod_{X \in \mathscr{H}}\left(X_{E}, X^{E}\right)=\left(\bigcap_{X \in \mathscr{H}} X_{E},\left(\bigcap_{X \in \mathscr{H}} X_{E}\right) \bigcup\left(\bigcup_{X \in \mathscr{H}} B N_{E}(X)\right)\right) .
$$

Proof. Since $X_{E} \subseteq \bigcup_{X \in \mathscr{H}} X_{E}$ and $\bigcap_{X \in \mathscr{H}} B N_{E}(X) \subseteq B N_{E}(X)$, for each $X \in \mathscr{H}$, we have $\left(X_{E}, X^{E}\right) \sqsubseteq\left(\bigcup_{X \in \mathscr{H}} X_{E},\left(\bigcup_{X \in \mathscr{H}} X_{E}\right) \cup\left(\bigcap_{X \in \mathscr{H}} B N_{E}(X)\right)\right)$ for all $X \in \mathscr{H}$, i.e. the right side is an upperbound for the system $\left\{\left(X_{E}, X^{E}\right) \mid X \in \mathscr{H}\right\}$. Now, suppose that $\left(Y_{E}, Y^{E}\right)$ is also an upper bound for $\left\{\left(X_{E}, X^{E}\right) \mid X \in \mathscr{H}\right\}$.

Then the relations $X_{E} \subseteq Y_{E}$ and $B N_{E}(Y) \subseteq B N_{E}(X)$, for all $X \in \mathscr{H}$ imply $\bigcup_{X \in \mathscr{H}} X_{E} \subseteq Y_{E}$ and $B N_{E}(Y) \subseteq \bigcap_{X \in \mathscr{H}} B N_{E}(X)$, proving that

$$
\left(\bigcup_{X \in \mathscr{H}} X_{E},\left(\bigcup_{X \in \mathscr{H}} X_{E}\right) \bigcup\left(\bigcap_{X \in \mathscr{H}} B N_{E}(X)\right)\right) \sqsubseteq\left(Y_{E}, Y^{E}\right) .
$$

This means that

$$
\left(\bigcup_{X \in \mathscr{H}} X_{E},\left(\bigcup_{X \in \mathscr{H}} X_{E}\right) \bigcup\left(\bigcap_{X \in \mathscr{H}} B N_{E}(X)\right)\right)
$$

equals to the supremum $\bigsqcup_{X \in \mathscr{H}}\left(X_{E}, X^{E}\right)$ of $\left\{\left(X_{E}, X^{E}\right) \mid X \in \mathscr{H}\right\}$. The second equality is proved dually.

Let $L$ be a complete lattice and an element $p \in L$. Then the set

$$
[p)=\{x \in L \mid x \geq p\}
$$

is called the principal filter belonging to $p$. In addition, we use the notion of an exact element of $R S(U, E)$ as follows:

$$
\left(X_{E}, X^{E}\right) \in R S(U, E) \text { is exact, if } X_{E}=X=X^{E} .
$$

\section{Corollary 3. The principal filter}

$$
[(\varnothing, \varnothing))=\{(X, Y) \in R S(U, E) \mid(\varnothing, \varnothing) \sqsubseteq(X, Y)\}
$$

is equal to the set of the exact elements of $R S(U, E)$.

Proof. Let $(X, X)$ be an exact element of the lattice $R S(U, E)$. Then clearly $(\varnothing, \varnothing) \sqsubseteq(X, X)$. Conversely, for any $(X, Y) \in[(\varnothing, \varnothing))$ the relation $(\varnothing, \varnothing) \sqsubseteq(X, Y)$ implies $Y \backslash X \subseteq \varnothing$, i.e. $X=Y$. This means that $(X, Y)$ is an exact element of $R S(U, E)$.

We give an example to illustrate the discussed structures. 
TABLE 1.

\begin{tabular}{|c||c|c||c|}
\hline$X$ & $X_{E}$ & $X^{E}$ & $B N_{E}(X)$ \\
\hline$\varnothing$ & $\varnothing$ & $\varnothing$ & $\varnothing$ \\
\hline$\{a\}$ & $\{a\}$ & $\{a\}$ & $\varnothing$ \\
\hline$\{b\}$ & $\{b\}$ & $\{b\}$ & $\varnothing$ \\
\hline$\{c\}$ & $\varnothing$ & $\{c ; d\}$ & $\{c ; d\}$ \\
\hline$\{d\}$ & $\varnothing$ & $\{c ; d\}$ & $\{c ; d\}$ \\
\hline$\{a ; b\}$ & $\{a ; b\}$ & $\{a ; b\}$ & $\varnothing$ \\
\hline$\{a ; c\}$ & $\{a\}$ & $\{a ; c ; d\}$ & $\{c ; d\}$ \\
\hline$\{a ; d\}$ & $\{a\}$ & $\{a ; c ; d\}$ & $\{c ; d\}$ \\
\hline$\{b ; c\}$ & $\{b\}$ & $\{b ; c ; d\}$ & $\{c ; d\}$ \\
\hline$\{b ; d\}$ & $\{b\}$ & $\{b ; c ; d\}$ & $\{c ; d\}$ \\
\hline$\{c ; d\}$ & $\{c ; d\}$ & $\{c ; d\}$ & $\varnothing$ \\
\hline$\{a ; b ; c\}$ & $\{a ; b\}$ & $U$ & $\{c ; d\}$ \\
\hline$\{a ; b ; d\}$ & $\{a ; b\}$ & $U$ & $\{c ; d\}$ \\
\hline$\{a ; c ; d\}$ & $\{a ; c ; d\}$ & $\{a ; c ; d\}$ & $\varnothing$ \\
\hline$\{b ; c ; d\}$ & $\{b ; c ; d\}$ & $\{b ; c ; d\}$ & $\varnothing$ \\
\hline$U$ & $U$ & $U$ & $\varnothing$ \\
\hline
\end{tabular}

Example 1. Consider a universe consisting of four elements $U=\{a, b, c, d\}$. Let $E$ be an equivalence relation on $U$ yielding the partitions

$$
U / E=\{\{a\},\{b\},\{c ; d\}\} .
$$

Table 1 contains the lower and upper approximations and the boundary regions for all subsets of $U$.

Based on Table 1 twelve different rough sets are detected and the rough set lattice with the inclusion order is presented in Figure 1. Using the boundary regions we construct the lattices $(B R S(U, E), \unlhd)$ and $(R S(U, E)$, ᄃ) and plot their Hasse diagrams in the next Figure 2. Comparing the diagrams of the lattices $(R S(U, E), \leq)$ (see Figure 1) and $(R S(U, E)$, $)$ (see Figure 2) their order-isomorphy is obvious, but the rough sets are located in different way in them.

As an illustration to Corollary 3 we list the exact rough sets from Table 1, which form the principal filter belonging to $(\varnothing, \varnothing)$ as follows:

$$
\begin{gathered}
{[(\varnothing, \varnothing))=\{(\varnothing ; \varnothing) ;(\{a\},\{a\}) ;(\{b\},\{b\}) ;(\{a ; b\},\{a ; b\}) ;(\{c ; d\},\{c ; d\}) ;} \\
(\{a ; c ; d\},\{a ; c ; d\}) ;(\{b ; c ; d\},\{b ; c ; d\}) ;(U, U)\} .
\end{gathered}
$$

The elements of the filter are the edges of the upper "cube" in the lattice $(R S(U, E)$, $)$ ) as it is visible in Figure 2. 


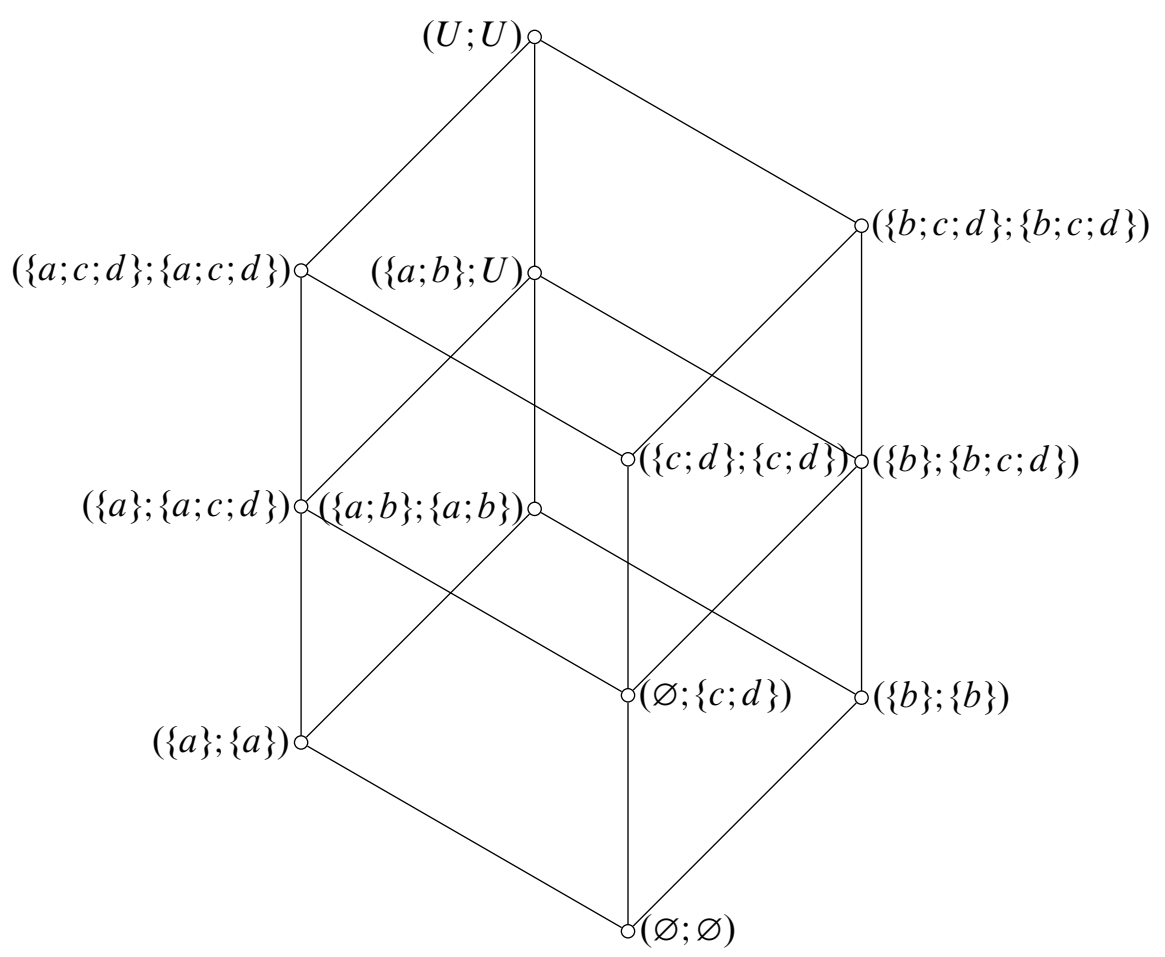

FIgURE 1. Forming rough sets and the rough set lattice $(R S(U, E), \leq)$ to the equivalence relation given in Example 1

\section{Conclusions}

Except for the properties of the well-known rough set lattice the order-theoretical consequences of rough set theory are less investigated. In this paper, starting from the traditional accuracy measure we propose a new order on the set of rough sets. This order induces a complete lattice which is isomorphic to the rough set lattice ordered by the inclusion relation. In order to prove the isomorphy of the structures we used a new type of representation of rough sets called boundary representation. For the sake of completeness the join and meet operators are also derived to this new lattice. We prove that the exact rough sets (for which the lower and upper approximations coincide) are specially located in our construction. To illustrate our examination a simple example is studied and the corresponding lattices are presented. As a future work we plan to explore further properties of this new order and study possible applications and examples. 


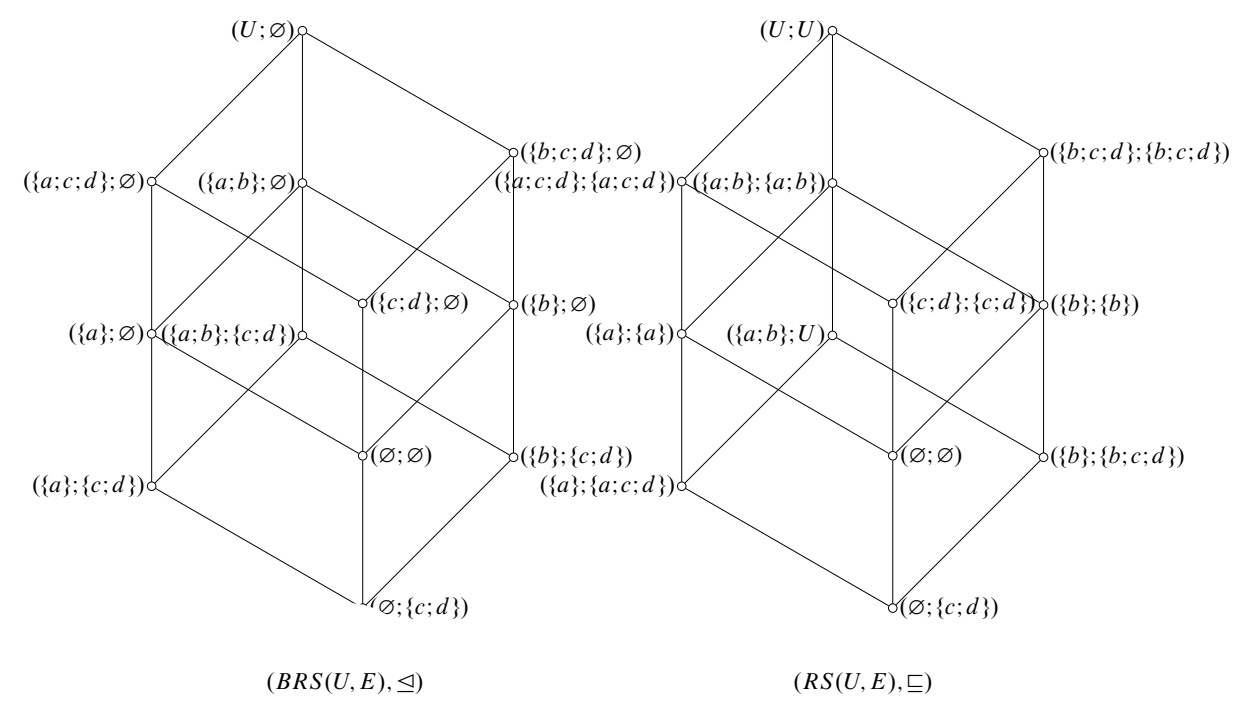

FIGURE 2. The Hasse diagram of the lattices $(B R S(U, E), \unlhd)$ and $(R S(U, E), \sqsubseteq)$

\section{REFERENCES}

[1] Belnap N.D., "A useful four-valued logic," in Modern Uses of Multiple-Valued Logic, J.M. Dunnand and G. Epstein, Ed. D.Reidel, 1977, pp. 8-37.

[2] Comer, S. D., "On connections between information systems, rough sets and algebraic logic," Algebraic Methods in Logic and Computer Science, Banach Center Publications, vol. 28, pp. 117-124, 1993, doi: 10.4064/-28-1-117-124.

[3] Ganter, B. and Wille, R., Formal Concept Analysis, Mathematical Foundations. Berlin: SpringerVerlag, 1999. doi: 10.1007/978-3-642-59830-2.

[4] Grätzer, G., General Lattice Theory, 2nd ed. Basel: Birkhäuser, 1998. doi: 10.1007/978-30348-7633-9.

[5] Järvinen, J., "Approximations and rough sets based on tolerances," in Rough Sets and Current Trends in Computing 2000, Ziarko, W. and Yao, Y. Y., Ed. Berlin, Heidelberg: Springer, 2001, pp. 182-189, doi: 10.1007/3-540-45554-X-21.

[6] Mousavi, A., Jabedar-Maralani, P., "Relative sets and rough sets," International Journal of Applied Mathematics and Computer Science, vol. 11, pp. 637-653, 2001.

[7] Pagliani, P., "Rough set systems and logico-algebraic structures," in Incomplete Information: Rough Set Analysis, Orlowska, E., Ed. Heidelberg: Physica, 1997, pp. 109-190, doi: 10.1007/978-3-7908-1888-8-6.

[8] Pawlak, Z., “Rough sets,” Int. J. Comput. Inf., vol. 11, pp. 341-356, 1982, doi: 10.1007/BF01001956.

[9] Pomykala, J. and Pomykala, J. A., "The Stone algebra of rough sets," Bull. Polish Acad. Sci. Math., vol. 36, no. 7-8, pp. 495-508, 1988. 
[10] Wang, J., Liang, J., Qian, Y. and Dang, C., "Uncertainty measure of rough sets based on a knowledge granulation for nincomplete information systems," International Journal of Uncertainty, Fuzziness and Knowledge-Based Systems, vol. 16, no. 02, pp. 233-244, 2008, doi: 10.1142/S0218488508005157.

[11] Xu, B., Zhou, Y and Lu, H., "An improved accuracy measure for rough sets," Journal of Computer and System Sciences, vol. 71, no. 2, pp. 163-173, 2005, doi: 10.1016/j.jcss.2005.02.002.

[12] Yao, Y. Y., "Information granulation and rough set approximation," International Journal of Intelligent Systems, vol. 16, no. 1, pp. 87-104, 2001, doi: 10.1002/1098-111X(200101).

[13] Yao, Y. Y., "Notes on rough set approximations and associated measures," Journal of Zheijang Ocean University (Natural Science), vol. 29, no. 5, pp. 399-410, 2010.

[14] Yao Y.Y., Wong S.K.M., Lin T.Y., "A Review of Rough Set Models," in Rough Sets and Data Mining. Boston, MA: Springer, 1997, pp. 47-75, doi: 10.1007/978-1-4613-1461-5-3.

Authors' addresses

Attila Körei

Institute of Mathematics, University of Miskolc, 3515, Hungary

E-mail address: matka@uni-miskolc.hu

Sándor Radeleczki

Institute of Mathematics, University of Miskolc, 3515, Hungary

E-mail address: matradi@uni-miskolc.hu

Szilvia Szilágyi

Institute of Mathematics, University of Miskolc, 3515, Hungary

E-mail address: matszisz@uni-miskolc.hu 